\title{
Structural and Parametric Analysis of Robust Design Quality of Complex Technical Systems
}

\author{
Aleksandr Dmitriev ${ }^{1,2}$, Tatyana Mitroshkina ${ }^{1, *}$ and Gennady Rogachev ${ }^{3}$ \\ ${ }^{1}$ Samara National Research University named after academician S.P. Korolev (Samara University), 443086 Samara, Russia \\ ${ }^{2}$ Samara Region State Academy (Nayanova), 443010 Samara, Russia \\ ${ }^{3}$ Samara State Technical University, 443100, Samara, Russia
}

\begin{abstract}
Discussed the approaches to robust design of complex technical systems based on Quality Function Deployment method QFD. It demonstrates how to use the matrix approach to the implementation of QFD, which enables the structural and parametric analysis of the quality of complex technical systems in conditions of uncertainty and ill-conditioning problem
\end{abstract}

\section{Introduction}

Effective quality design of complex technical systems based on sustainable design and methods of quality management - the basis of improving the competitiveness of products and enterprises [1-4].

The aim of this work is to improve the quality of complex engineering systems at the design stage. It is proposed to use a robust design based on modern methods of quality management, as well as approaches to the structural and parametric identification and optimization [1-6].

Product design plays a crucial role in planning and providing quality products and services. It should be noted that the evolution of quality management techniques aimed to develop effective measures against unwanted volatility. Work on the genitive Taguchi robust design is the key to this evolution [4].

The purpose of the robust approach to product design - to make the output characteristics of the product low sensitivity to environmental variability, degradation products and manufacturing imperfections. Improved product design and manufacturing process, it allows to reduce production costs and product operation.

Taguchi divided the life cycle of any commodity product into two unequal parts [4]. First - the development and implementation of products everything that precedes the production of the product. The second - the actual production and operation. quality problems should start to engage in the first stage and the earlier, the better. It was then that it is possible not to tighten control and use a very committed, very expensive equipment, and establish a process for the design and production so that its characteristics were the least affected by the scatter / variability due to the imperfections of the manufacturing process variability of environmental conditions, heterogeneity of raw materials and other factors that are unavoidable in the manufacture and maintenance of products.
Taguchi proposes to divide the factors into groups so that one of them turned out to be the factors responsible for the primary response ("nominal"), and in another - are responsible for the spread. To identify these groups Taguchi introduces a new generic response that he calls "the signal / noise ratio." The original problem is divided into two independent optimization procedures: one for the denomination, and the other for variation.

Taguchi has developed an approach to the establishment of the nominal product and process parameters: system design and parametric design. System design - the process of the application of scientific and engineering knowledge to the development of the product structure model. The model defines the initial values of parameters of the product (or process). System design involves consideration of both customer requirements and production conditions. Robust parametric design - the process of identifying such values parameters of the product (or process), which reduce the sensitivity to design parameters change sources. The use of non-linear effects of parameters of product (or process) on the output parameters to reduce the sensitivity of Engineering to scatter sources is the essence of parametric design.

\section{Robust design}

In the arsenal of modern methods of transformation of customer requirements in the immediate characteristics of the new (or upgraded) product quality identification method include the quality function deployment QFD [2]. In expanded form, the process of implementing QFD includes four levels, covering all stages from conceptual design (system) design products and processes before deciding on the need to change the target parameters of manufacturing processes. On the technical level I determined (system) product characteristics, ensuring the implementation of customer requirements. Then deployed specifications / characteristics of the components are structured to product (QFD level 1, QFD level 2), further process parameters are determined that achieve the

"Corresponding author: t.mitroshkina@gmail.com 
desired components and characteristics values of the product (QFD level 3), and then specifies the requirements for execution of operations in the production of (QFD level 4).

The aim of the method is the transformation of the consumer request (needs and expectations) in the technical product specifications and work instructions, visualization, documentation and planning quality. The main element of QFD is precisely the deployment of customer requirements in the production and the attainment of the relevant technical specifications that meet the expectations of the consumer. In other words, in this case it comes to the production of the preparations for the launch of a new product.

At each of the levels of QFD developed a special table, called the House of Quality (HoQ). The result of the application of QFD is to define the priorities and identifying the characteristics of products and components, process parameters and production operations, the greatest impact on the realization of customer requirements.

The traditional solution quality planning tasks products and services within the QFD method is a consistent ranking of performance in accordance with this priority (result), considering the importance of customer requirements (raw data).

The absolute priority value characteristics $\Theta_{j}$ at QFD level 1 is calculated as follows:

$$
\Theta_{i}=\sum_{i=1}^{i=k}\left(P_{i} \cdot H_{i j}\right),
$$

where $H_{i j}$ - the coefficient of correlation characteristics $j$ and $i$ requirements, $P_{i}-$ the importance of the $i$ requirement for the consumer; $k$ - the number of customer requirements; $n$ - number of characteristics.

Characteristics are ranked by descending priority and relative value determined by the characteristics requiring clarification first. This model is the same for payments in the implementation of the method at all levels of QFD.

In the traditional approach the complexity and bulkiness of manual calculations combined with the absence of error accounting source data, resulting in low stability and reliability of the results $[1,3]$.

The approach to parametric identification of characteristics of complex technical systems (QFD level 1, QFD level 2) and process parameters (QFD level 3, QFD level 4), based on the matrix approach can significantly simplify the solution of practical problems of planning quality and improve the accuracy and robustness of the results to errors in the original data.

\section{Structural analysis}

Objectification of the design lies in the structuring of the "voice" of the consumer, structuring and defining the technical characteristics of the relationship between the "voice" of the consumer and the technical characteristics. Typically, a mathematical model of the relationship is a correlation matrix. Further analysis is given of the correlation matrix or in the matrix formulation - based on linear mathematical model. The subsequent task of parametric identification of characteristics of complex technical system using QFD method requires the use of more information and a special approach to the solution. The existing model of such information as the relationship characteristics (often negative), the error in the preparation of marketing and benchmarking information, difficulties realization of modernization (no technology) is considered in the "manual" mode that prevents the use of the existing model of QFD in full as a tool to support decision solutions.

The problem is identifying the quality of feedback and incorrect in the mathematical sense: the space of the original data is not static, for the same basic source of data, there are many solutions, etc. The incorrectness is also evident in the high variability of the requirements in time, the ambiguity of the unspoken expectations, etc.

Due to the nature of the task (reverse, incorrect) and the modern facilities of computer processing of the results of proposed solutions for sustainable use of the method of approximate solution of the matrix equation (2), allowing reasonably consider a wide range of different additional information $[1,3]$.

$$
H \cdot \delta \Theta=\delta P,
$$

where $\delta \Theta-n$-dimensional vector of relative deviations of parameters of state (evaluation of the target values of the product characteristics for level 1 of QFD), $\delta P-k$ dimensional vector of relative deviations signs state (the importance of the requirements and expectations of consumers (QFD level 1), H - matrix of size $(k \times n)$ the coefficients of relationship requirements and characteristics (for QFD level 1), a linear mathematical model.

The specificity of ill-posed problems is the instability of their solution: small changes in the source data can cause large changes randomly making, that is, the error in the input data can play a crucial role. Solution of illposed problems is impossible without additional a priori information, which plays a key role.

It is proposed to use worked in science and engineering methods for solving ill-posed problems. Thus, by «identification» of generally understood as a set of methods for constructing mathematical models (mathematical description of the behavior) of the system or process. The problem of parametric identification is to estimate the unknown parameter vector per input and output signals [5-8].

\section{Parametric analysis}

To solve the practical problem of parametric identification of complex technical system performance target values of greatest interest are methods that allow resolving the ill-posed problem with a minimum of a priori information. One effective method for the approximate solution of such problems is the regularization method, which is based on the concept of a regularizing algorithm [8].

Due to the nature of the mathematical formulation of the problem of identification of quality and modern computer capabilities are encouraged to use sustainable method for the approximate solution of the matrix equation based on QFD [1, 3]. The method can 
reasonably be considered in addition to interconnection requirements and characteristics of additional information: the relative changes in state parameters (change the values of the product characteristics), the relative changes in the status indication (change of degree of compliance with the requirements and expectations of consumers) $[1,3]$.

Creating a sustainable method of identifying the mathematical model in the system design and production processes is an important task. This is primarily since the traditional identification methods (for example, using the least squares method OLS) often do not provide reliable results under practical conditions of use.

It is known that the accuracy of the OLS estimator is characterized by a covariance matrix $\left(H^{T} P H\right)^{-1}$. Let

$$
A=\left(H^{T} P H\right)
$$

It seems appropriate to explore parametric characteristics of the matrix to allow further study of the stability (robustness) the resulting estimates.

The following harmonized standards are commonly used:

$$
\begin{gathered}
\|A\|_{1}=\max _{i}\left(\sum_{j}\left|a_{i j}\right|\right),\|A\|_{2}=\max _{j}\left(\sum_{i}\left|a_{i j}\right|\right), \\
\|A\|_{3}=\sqrt{\max _{j} \lambda_{A A^{T}}^{i}} .
\end{gathered}
$$

where $\lambda_{A A^{T}}^{i}$ - the eigenvalues of the matrix $A A^{T}$.

An important characteristic parameter matrix $A$ is the number of conditionality:

$$
\rho(A)=\max _{i} \lambda^{i} / \min _{i} \lambda^{i}
$$

where $\lambda^{i}$ - eigenvalues $A$.

The study was conducted on the model of "turboshaft aircraft engine with free turbine TV 7-117A" complex technical system. It is based on a linear mathematical model (LMM) containing the factors of influence on the maximum continuous operation.

Abstract norm of the inverse matrix of normal equations and the condition number of the inverse matrix of normal equations during statistical tests (the first 20 trials). The experiment was conducted by applying to the calculated values of the linear mathematical model of random variables distributed by the normal distribution in the range of $\pm 20 \%$. This variability LMM coefficients determined linearization errors, and the uncertainty of the initial state.

Results on the change of a matrix normal equations $\rho$ depending on the mathematical model of random errors.

These results show the values of the norm of the inverse matrix of normal equations and the condition number of the inverse matrix of normal equations in the statistical tests (the first 20 trials).

Statistical data obtained after the processing results are presented in Table 1.

Similarly, research conducted for a bypass engine. It can be concluded that the picture quality in the test result is the same. However, from a quantitative point of view, the value of the sample variance in TV 7-117A study have smaller values in almost all similar cases. Ego can be explained by the fact that the original version of LMM TV 7-117A has a matrix of normal equations, not as bad as the original version due to bypass engine. In addition, the criteria of a matrix of normal equations for the LMM with superimposed random errors for TV 7-117A perform better on average than TV 7-117A. Ego again confirms the conclusion about the impact on the error status parameter estimates matrix structure of normal equations, in particular, its condition number.

Table 1. Statistical characteristics of the rules of the inverse matrix of normal theater TV 7-117A equations for modeling the original matrix $H$

\begin{tabular}{|c|c|c|}
\hline $\begin{array}{c}\text { Statistical } \\
\text { characteristics }\end{array}$ & $\begin{array}{c}\text { For the inverse } \\
\text { matrix of the } \\
\text { matrix norm } \\
\left\|A^{-1}\right\|\end{array}$ & $\begin{array}{c}\text { For the condition } \\
\text { number } \rho\end{array}$ \\
\hline Average & 0,896 & 11800 \\
\hline $\begin{array}{c}\text { The standard } \\
\text { deviation }\end{array}$ & 0,322 & 4148,05 \\
\hline $\min$ & 0,439 & 5500 \\
\hline $\max$ & 1,688 & 21500 \\
\hline
\end{tabular}

\section{Discussion}

Using matrix approach to the implementation of QFD allows for structural and parametric analysis of the quality of complex technical systems in conditions of uncertainty and poor conditioning of the problem and be the basis of robust design. The quality of robust design proposed to estimate using the inverse matrix and the number of conditionality rules.

\section{References}

1. A.Ya. Dmitriev, T.A. Mitroshkina, Ontology of designing, 3(17), 313-327 (2014) [In Rus]

2. Y. Akao, G.H. Mazur, International Journal of Quality \& Reliability Management, 20(1), 20-35 (2003)

3. A. Dmitriev, T. Mitroshkina. ITM Web of Conferencesm, 6, 02005 (2016)

4. R.N. Kackar, A.C. Shoemaker, AT\&T Technical Journal 65(2), 39-50 (1986)

5. K.J. Astrom, P. Eykhoff. Automatica, 7, 123-162, (1971)

6. L. Ljung. System identification. Theory for the User, (PTR Prentice Hall, 1999)

7. E. Pluzhnik, E. Nikulchev, Virtual Laboratories in Cloud Infrastructure of Educational Institutions, 2014 2nd International Conference on Emission Electronics (ICEE), 67-69 (2014)

8. S.K. Bochkarev, A.Y. Dmitriev, V.V. Kulagin et al. Izvestiya Vysshikh Uchebnykh Zavedenii Aviatsionaya Tekhnika, 2, 68-70 (1993) [In Rus] 